Gefässchirurgie 2009 · 14:388-390

DOI 10.1007/s00772-009-0677-1

Online publiziert: 13. März 2009

๑) Springer Medizin Verlag 2009
A. Neumann · V. Göber · S. Schenker · J. Schmidli

Klinik für Herz- und Gefässchirurgie, Inselspital Bern

\title{
Invalidisierende Kalziphylaxie (Calcific uraemic arteriolopathy)
}

\section{Anamnese, klinischer Befund}

Ein 45-jähriger selbstständiger Unternehmer leidet seit seinem 11. Lebensjahr an Diabetes mellitus Typ 1. Dieser führte zu einer diabetischen Nephropathie, die im Alter von 35 Jahren dialysepflichtig wurde. Zwei Jahre später bekam der Patient eine Nierentransplantation. Im Alter von 42 Jahren traten schmerzhafte Ulzerationen an den Fingerspitzen beider Hände auf (• Abb. 1). Diese ließen sich konservativ nicht behandeln. Es kam zu bakteriellen Superinfektionen und Nekrosebildungen. In der Folge waren Amputationen, initial der Kleinfingerendglieder, notwendig.

\section{Diagnose, Therapie, Verlauf}

Anhand des dermatologischen Bildes und der Klinik wurde die Diagnose einer Kalziphylaxie gestellt. Bei erhöhtem Parathormonspiegel, bedingt durch einen tertiären Hyperparathyreoidismus, der medikamentös nicht zu senken war, wurde eine Parathyreoidektomie mit Autotransplantation in die Tibialis-anterior-Loge rechts durchgeführt. Der Patient wurde anschließend mit Sildenafil behandelt und erhielt engmaschige Kontrollen des Kalzium-Phosphat-Spiegels.

Dennoch kam es immer wieder zu Ulzerationen an beiden Händen und Füssen, die dem Patienten stärkste Schmerzen bereiteten. An den Händen mussten die Finger teilamputiert (• Abb. 2) wer- den, ebenso der rechte Unterschenkel und der Vorfuß links.

Weiterhin leidet der Patient an einer hypertensiven und koronaren Kardiopathie sowie einer Hyperlipoproteinämie und rezidivierenden Gichtarthritiden. Es erfolgten eine perkutane transluminale koronare Angioplastie und Stentimplantation in die rechte Koronararterie und in den Ramus interventricularis anterior.

Aktuell erfolgt die medikamentöse Therapie mit Acetylsalicylsäure, Irbesartan, Torasemid, Pravastatin, Calcitriol, Calciumacetat, Prednison, Mycophenolsäure, intensivierter Insulinbehandlung und Darbepoetin. Im Rahmen der Schmerztherapie werden Paracetamol, transdermales Fentanyl und Morphintropfen verabreicht.

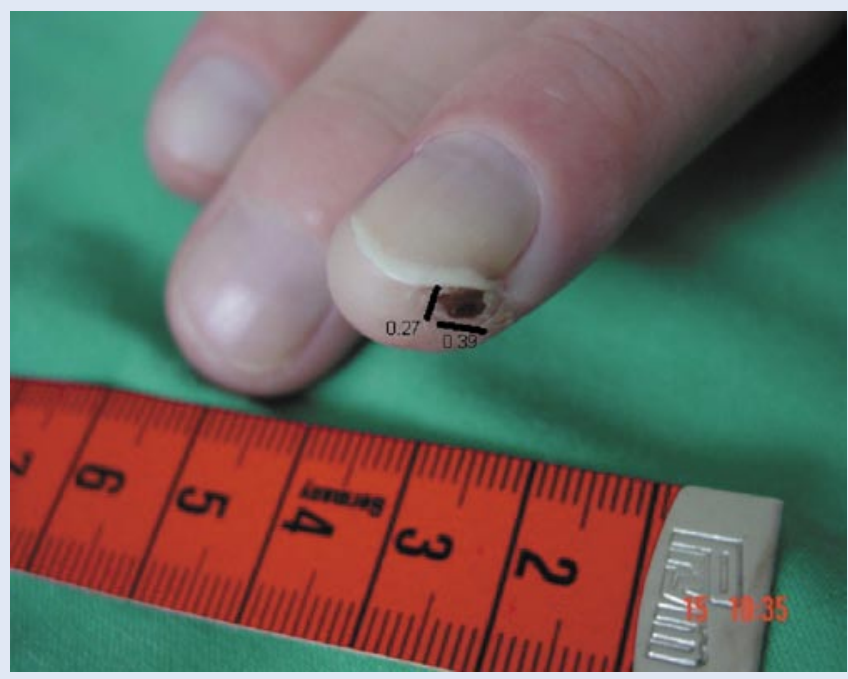

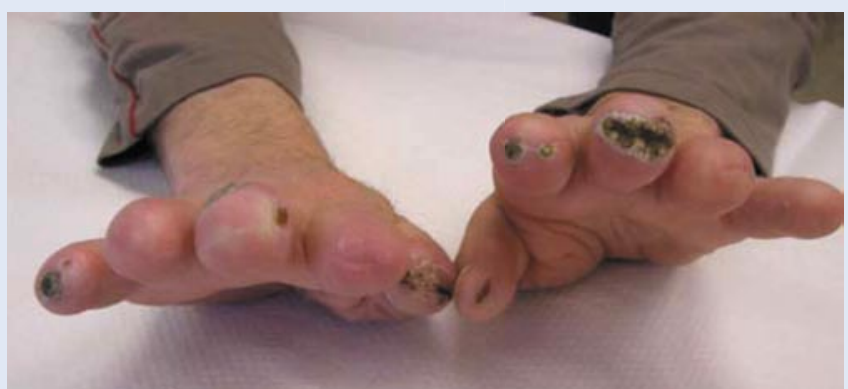

Abb. $2 \triangle$ Zustand nach Fingerteilamputationen aufgrund stark schmerzhafter Ulzerationen mit Nekrosebildungen

Abb. $1<$ Schmerzhafte Ulzeration am Zeigefinger rechts 


\section{Diskussion}

Die Kalziphylaxie ist eine Erkrankung, die 1-5\% aller Patienten mit mehr als einjähriger Dialysedauer betrifft. Die Inzidenz während der vergangenen 10 Jahre ist steigend [7]. Es wurden aber auch FäIle ohne Vorliegen einer Niereninsuffizienz beschrieben, so z. B. im Rahmen eines paraneoplastischen Syndroms bei Mammakarzinom, bei primärem Hyperparathyreoidismus oder bei chronisch entzündlichen Darmerkrankungen. Die Krankheit befällt mehr Frauen als Männer [4].

Erstmals beschrieben wurde das Krankheitsbild 1898 von Bryant und White. Seyle et. al [8] postulierten 1962 als eine mögliche Ursache der Kalziphylaxie sog. Sensitizer, Stoffe, die eine Kalziummobilisation verursachen (Vitamin D, Parathormon, erhöhtes Kalzium-Phosphat-Produkt) und Challenger, Stoffe, die in Kombination mit den Sensitizern eine Entzündungsreaktion provozieren können (Protein-C-Mangel). Bei der Therapie von Kalziphylaxiepatienten sollten Substanzen aus beiden Gruppen vermieden werden.

Die Krankheit manifestiert sich primär in kutanen Läsionen, die bei 90\% der Patienten symmetrisch an den unteren Extremitäten auftreten [1]. Initial bilden sich livide Erytheme, aus denen sich im Verlauf schmerzhafte Ulzerationen und $\mathrm{Ne}$ krosen entwickeln.

Histologisch findet man eine Intimaproliferation und eine Mediakalzifizierung der kleinen Gefäße. Besonders betroffen sind die Kapillaren, Venolen und Arteriolen der Dermis und der Subkutis. Die Intimahyperplasie führt zu einer Okklusion des Gefäßlumens und kann so eine sekundäre Thrombenbildung begünstigen.

Auf dem Boden der Ulzerationen entwickeln sich häufig therapierefraktäre Superinfektionen, die bis zur Sepsis führen können und die hohe Letalität von 80\% erklären [7].

Die Diagnosestellung erfolgt anhand der Klinik und des dermatohistopathologischen Bildes. Empfohlen wird die Entnahme einer Biopsie an den betroffenen Stellen. Jedoch wird vor Stichprobenfehlern und Initiierung oder Verschlechterung ulzeröser Läsionen gewarnt.
Die Ätiologie der Erkrankung ist nicht vollständig geklärt. Es werden sowohl Störungen im Kalzium-Phosphat-Haushalt als auch ein Fehlen oder vermindertes Vorhandensein homöostatischer Mechanismen diskutiert [6].

Es existiert zur Zeit keine kausale Therapie. Kalziphylaxiepatienten müssen aufgrund der sehr therapieresistenten Ulzerationen im Verlauf vielfache Amputationen über sich ergehen lassen.

Es gibt verschiedene Therapieansätze mit dem Ziel der optimalen Kalziumund Phosphatkontrolle, die zu einer Besserung oder vollständigen Abheilung der Ulzera geführt haben. Beim Vorliegen eines Hyperparathyreoidismus wurden deutliche Verbesserungen der Kalziumund Phosphatkontrolle sowie der Wundheilung nach Parathyreoidektomie beschrieben [5]. In einem Fallbericht wurde erfolgreich mit niedrig dosiertem Plasminogenaktivator behandelt. Bei diesem Patienten kam es innerhalb von 6 Monaten zur kompletten Abheilung aller Ulzera [9].

Intravenöse oder intraperitoneale Infusion des Chelatbildners Natriumthiosulfat zeigten gute Resultate in der symptomatischen Behandlung [3, 10].

Ein weiterer Therapieansatz zur Verbesserung der Wundheilung und Verminderung der Mortalität besteht in der Anwendung einer hyperbaren Sauerstofftherapie [2]. Gefäßchirurgische Revaskularisationsversuche brachten aufgrund der geringen Zielgefäßgröße keine zufriedenstellenden Ergebnisse.

\section{Fazit für die Praxis}

Die Kalziphylaxie ist ein seltenes Krankheitsbild, das primär niereninsuffiziente, dialysepflichtige Patienten befällt, aber auch im Rahmen anderer Grunderkrankungen beschrieben wird. Die Krankheit manifestiert sich in kutanen, therapierefraktären Hautläsionen und geht mit einer hohen Mortalität einher. Es existiert keine kausale Therapie. Empfohlen werden supportive Maßnahmen wie ein adäquates Wundmanagement und eine optimale Kalzium- und Phosphatkontrolle.
Gefässchirurgie 2009 · 14:388-390

DOI 10.1007/s00772-009-0677-1

c) Springer Medizin Verlag 2009

A. Neumann · V. Göber · S. Schenker . J. Schmidli Invalidisierende Kalziphylaxie (Calcific uraemic arteriolopathy)

Zusammenfassung

Ein 45-jähriger Patient mit langjährigem Diabetes mellitus Typ 1 und diabetischer Nephropathie stellt sich vor. Nach zweijähriger Hämodialyse erfolgte im Alter von 35 Jahren eine Nierentransplantation. Aufgrund des klinischen Verlaufs mit rezidivierenden akralen Nekrosen wurde die Diagnose Kalziphylaxie gestellt. Im weiteren Verlauf erfolgten mehrfach Amputationen im Bereich der oberen und unteren Extremitäten. Die Pathogenese, das klinisches Bild und die Behandlung der Kalziphylaxie werden im Artikel besprochen.

Schlüsselwörter

Kalziphylaxie · Calcific uraemic arteriolopathy · Niereninsuffizienz $\cdot$ Hämodialyse $\cdot$ Kutane Nekrosen

Disabling calciphylaxis (calcific uraemic arteriolopathy)

Abstract

We present the case of a 45-year-old man with diabetes mellitus type 1 and end-stage renal failure who was treated with haemodialysis for 2 years and received a renal transplant at the age of 35 . Due to clinical progression with recurrent acral necrosis, the diagnosis of calciphylaxis was made. Over the course of time, many amputations on both hands and legs were necessary. The pathogenesis, clinical features, diagnosis, and treatment of calciphylaxis are discussed.

\section{Keywords}

Calciphylaxis - Calcific uraemic arteriolopathy · Renal failure · Hemodialysis - Cutaneous necrosis 


\section{Korrespondenzadresse}

\section{Dr.V.Göber}

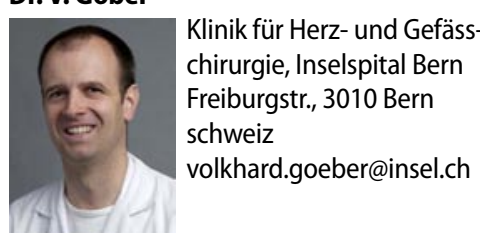

Interessenkonflikt. Der korrespondierende Autor gibt an, dass kein Interessenkonflikt besteht.

\section{Literatur}

1. Angelis M, Wong LL, Meyers SA, Wong LM (1997) Calciphylaxis in patients on hemodialysis: a prevalence study. Surgery 122:1083-1089

2. Arenas MD, Gil MT, Gutiérrez MD et al (2008) Management of calcific uremic arteriolopathy (calciphylaxis) with a combination of treatments, including hyperbaric oxygen therapy. Clin Nephrol 70(3):261-264

3. Brucculeri M, Cheigh J, Bauer G, Serur D (2005) Long-term intravenous sodium thiosulfate in the treatment of a patient with calciphylaxis. Semin Dial 18:431-434

4. Fine A, Zacharias J (2002) Calciphylaxis is usually nonulcerating: risk factors, outcome and therapy. Kidney Int 61:2210-2217

5. Hafner J, Keusch G, Wahl C et al (1995) Uremic small-artery disease with medial calcification and intimal hyperplasia (so-called calciphylaxis): a complication of chronic renal failure and benefit from parathyroidectomy. J Am Acad Dermatol 33:954-962

6. Levin A, Metha RL, Goldstein MB (1993) Mathematical formulation to help identify the patient risk of ischemia tissue necrosis - a potentially lethal complication of chronic renal failure. AM J Nephrol 13:448-453

7. Rogers NM, Coates PT (2008) Calcific uraemic arteriolopathy: an update. Curr Opin Nephrol Hypertens 17(6):629-634

8. Seyle H, Gabbiani G, Strebel R (1962) Sensitization to calciphylaxis by endogenous parathyroid hormone. Endocrinology 71:554-558

9. Sewell LD, Weenig RH, Davis MDP et al (2004) Low dose tissue plasminogen activator for calciphylaxis. Arch Dermatol 140:1043-1048

10. Tindni A, Gaurav K, Panda M (2008) Non-healing painful ulcers in a patient with chronic kidney disease and role of sodium thiosulfate: a case report Cases J 1(1):178

\section{R. Larsen}

\section{Anästhesie und Intensivmedizin in der Herz-, Thorax- und Gefäß- chirurgie}

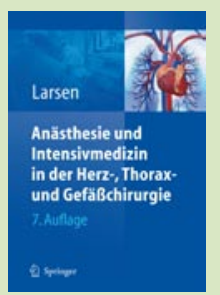

Heidelberg: Springer

Medizin Verlag 2009

458 S., 94 Abb.,

(ISBN 13 978-3-540-

88794),

64.00 EUR

„Anästhesie und Intensivmedizin in der Herz-, Thorax- und Gefäßchirurgie" lautet der Titel des vorliegenden Fachbuchs, das von Reinhard Larsen verfasst wurde und nun bereits in der 7. aktualisierten und überarbeiteten Auflage vorliegt. Dieses eindeutig klinisch ausgelegte Lehrbuch umfasst 458 Seiten, ist in 15 Kapitel gegliedert und befasst sich mit den anästhesiologischen und intensivmedizinischen Aspekten der Fachgebiete „HTG", also der Herzchirurgie, Thoraxchirurgie und Gefäßchirurgie, wobei erwartungsgemäß der Bereich "Herzchirurgie" mit 340 Seiten den größten Teil ausmacht.

In den ersten 5 Kapiteln werden die Grundlagen besprochen: zuerst alle Anästhetika und Adjuvanzien, wobei hier großer Wert auf das Verständnis der teilweise komplexen kardio-vaskulären Eigenschaften der Substanzen gelegt wird, dann die eigentlichen kardiovaskulären Medikamente - hier erfährt der Leser alle Details, die für einen differenzierten Umgang mit diesen Substanzen erforderlich sind. Anschließend werden die verschiedenen Monitoringverfahren der Herz-Kreislauf-Funktion und ihre sinnvolle Interpretation dargestellt: arterielle, zentralvenöse und pulmonalarterielle Drücke, aber auch PICCO, LAP und TEE. Anschließend werden Aufbau und Funktionsweise der Herzlungenmaschine (HLM) beschrieben und dann - für die praktische Tätigkeit von hoher Relevanz - das anästhesiologische Management bei Patienten, die an die HLM angeschlossen werden: Wie genau wird kanüliert, welche Bypassart in welcher Situation, was ist konkret zu beachten beim Anschluss und beim Abgehen von der HLM?

Die nächsten 6 Kapitel beschäftigen sich mit den verschiedenen herzchirurgischen Erkrankungen, ihren typischen Operationen und der dazu gehörenden anästhesiologischen und intensivmedizinischen Versorgung: KHK und die aortokoronare Bypassoperation, die verschiedenen Herzklappenvitien und ihre Operationen, die Herztransplantation, ein ausführliches und gut verständliches Kapitel zu dem immer etwas schwierigeren Thema der kongenitalen Herzfehlern und ihren Korrekturoperationen und abschließend ein Kapitel zu Anästhesie und Intensivtherapie bei Herzschrittmachern und Kardiovertern bzw. Defibrillatoren.

Die verbleibenden 4 Kapiteln runden das Thema ab: Ausführliches zu Anästhesie und Intensivmedizin bei Operationen an Lunge und Trachea, jeweils ein Kapitel zur Versorgung von Patienten mit thorakalem bzw. abdominellem Aortenaneurysma sowie mit Karotisstenose.

Das gesamte Buch ist hervorragend strukturiert; der Text selbst ist - wie man es von einem „Larsen“ nicht anders erwartet - sprachlich gut aufbereitet, verständlich und eindeutig auf die klinische Anwendung ausgerichtet; das Literaturverzeichnis zu den einzelnen Kapiteln enthält die aktuellen und relevanten Veröffentlichungen zu den jeweiligen Themen.

Wenn es überhaupt eine Kritik gibt, so sei angemerkt, dass der Abschnitt Echokardiographie und TEE sehr knapp ausgefallen ist. Eine umfassende Darstellung würde sicherlich ein eigenes Lehrbuch erfordern, doch eine etwas ausführlichere Beschreibung mit typischen Bildbefunden würde das Monitoringkapitel abrunden - dies wäre mein Wunsch für die nächste Auflage.

Zusammenfassend ist es dem Autor in hervorragender Weise gelungen, den in der Herz-, Thorax- und Gefäßchirurgie tätigen Ärztinnen und Ärzten ein umfassendes und dabei trotzdem praxisorientiertes Lehrbuch an die Hand zu geben, das ausführlich nahezu alle Fragen beantwortet, die bei der Planung und Durchführung von Anästhesie und Intensivmedizin bei Eingriffen in der Herz-, Thorax- und Gefäßchirurgie auftreten können. Allen Anästhesistinnen und Anästhesisten, die im Bereich „HTG" arbeiten oder sich auf einen Einsatz dort vorbereiten wollen, sei die Lektüre dieses Buches ausdrücklich empfohlen.

Professor Wolfram Wilhelm (Lünen) 